\title{
THE STABILITY OF A DIRECT METHOD FOR SUPERRESOLUTION
}

\author{
José M. N. Vieira and Paulo J. S. G. Ferreira \\ Dep. de Electrónica e Telecomunicações \\ Universidade de Aveiro \\ 3810 Aveiro, Portugal \\ E-mail: pjf@inesca.pt
}

\begin{abstract}
A direct method for superresolution recently proposed by Walsh and Delaney is further analyzed from the point of view of numerical stability. The method is based on a set of linear equations $A x=b$, where $A$ is $m \times n$, and $b$ is a subset (of cardinal $n$ ) of the Fourier transform of the object (which has a total of $N$ samples). We give exact and best possible approximate expressions for the determinant of $A$, when $m=n$. As a corollary, it is shown that the smallest eigenvalue of $A$ in absolute value satisfies $\left|\lambda_{\min }\right| \leq k(n) N^{-(n-1) / 2}$, where $k(n)$ (which is independent of $N$ ) is explicitly given. The magnitude of the smallest eigenvalue of $A$ becomes increasingly small as $N$ grows, even when the number of unknowns $n$ remains constant. When $m>n$ the singular values of $A$ are studied, and related to the eigenvalues of the matrix of two other direct methods. As a result, the connection between the method and the other direct methods is clarified.
\end{abstract}

\section{INTRODUCTION}

It is quite well-known that the resolution of a time-domain or space-domain object can be improved by restoring lost frequency-domain information, a process that is known as superresolution.

The theory and algorithms that relate to this problem use the available information concerning the object in both the space-domain and the Fourier domain. As an example, the object, mathematically modeled by a function $x$, might be finite, and its extent is usually known. For example, the support of the function $x$ might be a certain given compact set. On the other hand, its Fourier transform $\hat{x}$ might be partially known (in the sense that $\hat{x}(\omega)$ is known for some values of $\omega$ ). Under these two hypotheses, it is theoretically possible to estimate the object given the available information only (in other words, it is possible to extrapolate the Fourier transform of the object).

This work was supported by JNICT.
The existence and uniqueness of the solution to this superresolution / extrapolation problem is a straightforward consequence of the analyticity of the Fourier transform of a compactly supported object. However, analytic continuation does not seem to lead to any practical superresolution algorithms, and other solutions are needed.

One of the first methods proposed uses the double orthogonality of the prolate spheroidal functions [15]. Most other methods take advantage of the following facts: mathematically, the set of signals that satisfy the time or space domain conditions mentioned above (compact, known support) is a subspace of some parent Hilbert space $\mathcal{H}$ (often $L_{2}$ ). Similarly, the set of signals that satisfy the frequencydomain conditions defines another subspace of $\mathcal{H}$. Successive projections onto each of these subspaces lead to a sequence of approximations to the solution of the problem $[8,13,19]$. A more general alternative is the POCS (projections onto convex sets) method [14,20].

Numerical algorithms require discrete-time or discretespace formulations, that is, sampled data. The finite dimensional equivalent of the alternating projection or GerchbergPapoulis iteration was studied by Jones [11]. A more recent work [2] has pointed out several properties of some of the iterative methods in connection with the interpolation problem.

A number of noniterative methods have also been proposed. The one-step method introduced by Cadzow [1] is an example. The noniterative methods proposed by Gröchenig [10] and Ferreira [3], which at first glance appear to be based on rather distinct ideas, are in fact dual [5] in a certain sense. Recently, other methods have appeared $[9,12]$.

These works deal mostly with the signal extrapolation or interpolation problems. Specific results, in the light of [3], for the image reconstruction problem can be found in a variety of related works $[4,7]$. Strohmer $[16,17]$ has proposed efficient numerical algorithms (based on the preconditioned conjugate gradient method) to solve the signal and image extrapolation problem, starting from one of the direct methods [10]. 
Walsh and Delaney [18] have also given a direct (noniterative) solution to the discrete superresolution problem, which is based on a set of linear equations $A x=b$. It is the purpose of this paper to study the stability of their method and to relate it to the noniterative solutions described in some of the works mentioned above. More precisely, we give exact and best-possible bounds for the determinant of $A$, when $A$ is square. As a corollary we then show that $A$ has, in general, very small eigenvalues whose magnitude depend on the total number of samples of the data record $N$, and not only on the number of unknown samples. When the matrix is not square we show that its singular values are related to the eigenvalues of the direct methods proposed in $[3,10,17]$.

\section{THE METHOD}

Let $x \in \mathbb{C}^{N}$ denote a signal, and $\hat{x} \in \mathbb{C}^{N}$ its Fourier transform, defined by $\hat{x}=F x$, where $F$ is the $N \times N$ Fourier matrix with elements

$$
F_{a b}=\frac{1}{\sqrt{N}} e^{-\mathrm{j} \frac{2 \pi}{N} a b} .
$$

In the discrete superresolution problem [18] the signal $x \in$ $\mathbb{C}^{N}$ (with a total of $N$ samples $x_{0}, x_{1}, \ldots, x_{N-1}$ ) is known to vanish except for $n$ contiguous samples. Without loss of generality, the nonzero samples can be labeled

$$
x_{0}, x_{1}, \ldots, x_{n-1}
$$

Additionally, $m$ samples of the discrete Fourier transform $\hat{x}$ are known. The Fourier transform of a real signal $x \in \mathbb{R}^{N}$ satisfies $\hat{x}_{i}=\hat{x}_{N-i}^{*}$, and so one may assume without loss of generality that the known samples are

$$
\hat{x}_{0}, \hat{x}_{1}, \hat{x}_{2}, \ldots, \hat{x}_{k}, \hat{x}_{N-k}, \hat{x}_{N-k+1}, \ldots, \hat{x}_{N-1},
$$

with $m=2 k+1$.

As in [2], we denote by $E_{N}$ the set $\{0,1, \ldots, N-1\}$, and let $S_{a}$ and $S_{b}$ be two subsets of $E_{N}$ of $M$ elements each. We say that $S_{a}$ and $S_{b}$ are equivalent if the elements of $S_{a}$ can be obtained by addition of an integer constant, modulo $N$, to the elements of $S_{b}$. This means that $S_{a}$ and $S_{b}$ are related by a circular shift (a cyclic permutation). We say that a subset of $E_{N}$ of cardinality $M<N$ is contiguous if it is equivalent to $E_{M}=\{0,1, \ldots, M-1\}$.

It is clear that the indexes of the known Fourier transform samples in the superresolution problem, that is,

$$
\{0, \pm 1, \pm 2, \ldots, k\} \bmod N,
$$

are contiguous.

Mathematically, the discrete version of the superresolution problem is therefore a special case of the following problem: [5]
Problem 1 Let $S_{t}$ and $S_{f}$ be two proper nonempty subsets of $\{0,1, \ldots, N-1\}$. Given the sets $x\left(S_{t}\right):=\left\{x_{i}: i \in S_{t}\right\}$ and $\hat{x}\left(S_{f}\right):=\left\{\hat{x}_{i}: i \in S_{f}\right\}$, find $x$ and $\hat{x}$.

We will assume that $S_{t}$ and $S_{f}$ have $N-n$ and $m$ elements, respectively. In the superresolution problem the set $S_{t}$ is of the form

$$
S_{t}=\{n, n+1, n+2, \ldots, N-1\},
$$

and $x_{i}=0$ for all $i \in S_{t}$. On the other hand, the set $S_{f}$ can often be described as

$$
S_{f}=\{0,1,2, \ldots, k\} \cup\{N-k, N-k+1, \ldots, N-1\},
$$

where $m=2 k+1$. Generally speaking, one might have $S_{f}=\left\{i_{0}, i_{1}, \ldots, i_{m-1}\right\}$, a set of integers distinct modulo $N$. We will use this notation even when the elements of $S_{f}$ are contiguous modulo $N$, as in (1).

The solution proposed by Walsh and Delaney [18] is as follows. Consider the $N$ equations $\hat{x}=F x$, concentrating on the $m$ equations that correspond to known frequencydomain samples $\hat{x}_{i}$, that is,

$$
\hat{x}_{i}=\sum_{j=0}^{n-1} F_{i j} x_{j}, \quad\left(i \in S_{f}\right) .
$$

Note that $\bar{S}_{t}=\{0,1, \ldots, n-1\}$ and $x\left(S_{t}\right)=0$. We will use the notation $A x=b$, where $A_{j k}=F_{i_{j}}, b_{j}=\hat{x}_{i_{j}}$.

If $m \geq n$ one might hope to solve them for the $n$ unknown $x_{j}, 0 \leq j<n$. When the elements of $S_{f}$ and $S_{t}$ are not contiguous modulo $N$, this necessary condition is not sufficient (it is easy to exhibit examples of sets $S_{t}$ and $S_{f}$ such that the matrix $A$ has no inverse). But when either $S_{f}$ or $S_{t}$ are contiguous modulo $N$ the matrix $A$ will be Vandermonde, up to a scale factor, and consequently nonsingular. It is a bit less straightforward to show that contiguity is merely a sufficient condition for non-singularity.

\section{NUMERICAL STABILITY}

It is convenient to use the notation $S_{f}=\left\{i_{0}, i_{1}, \ldots, i_{m-1}\right\}$ despite the fact that we are considering a set $S_{f}$ given by (1). Assume that $m=n$. Then, as we have remarked, the matrix in (2) is square and proportional to a Vandermonde matrix,

$$
\hat{x}_{i_{k}}=\frac{1}{\sqrt{N}} \sum_{j=0}^{n-1} w^{i_{k} j} x_{j}, \quad(k=0,1, \ldots, m-1) .
$$

We have written $w=e^{-\mathrm{j} \frac{2 \pi}{N}}$. The determinant $\Delta$ of the matrix $A$ of this set of equations is given by

$$
\Delta=\frac{1}{N^{n / 2}} \prod_{j>k}\left(w^{i_{j}}-w^{i_{k}}\right)
$$


and consequently

$$
|\Delta|=\frac{1}{N^{n / 2}} \prod_{j>k}\left|w^{i_{j}}-w^{i_{k}}\right| .
$$

We will attempt to express the absolute value of this determinant in a more convenient form. Our task is slightly more simple if we note that the absolute value of the determinant is independent of a shift modulo $N$ in the set $S_{f}$. Consequently, we are free to assume without loss of generality that $i_{k}=k$.

The next step is to observe that the possible distances $\left|w^{i_{j}}-w^{i_{k}}\right|$ obtained as $j$ and $k$ range over all possible values $(j>k)$ are

$$
\left|1-w^{1}\right|,\left|1-w^{2}\right|,\left|1-w^{3}\right|, \ldots,\left|1-w^{n-1}\right|
$$

each distance being attained $n-1, n-2, \ldots, 1$ times, respectively. Therefore

$$
N^{n / 2}|\Delta|=\prod_{i=1}^{n-1}\left|1-w^{i}\right|^{n-i}
$$

or, recalling that $w=e^{-\mathrm{j} \frac{2 \pi}{N}}$,

$$
N^{n / 2}|\Delta|=\prod_{i=1}^{n-1}\left|2 \sin \left(\frac{\pi i}{N}\right)\right|^{n-i} .
$$

If $\pi i / N$ is small, the following approximation is valid:

$$
N^{n / 2}|\Delta| \approx \prod_{i=1}^{n-1}\left(\frac{2 \pi i}{N}\right)^{n-i}
$$

The closed form expression (3) is a convenient representation of the determinant, but we need to go one step further in order to estimate how small can the eigenvalues of $A$ be.

To probe further on the structure of (3) we need to expand the terms of the form $\sin (i x)$, with $x=\pi / N$, in terms of $\sin x$ and $\cos x$. This can be done as follows. First, setting $x=\pi / N$, note that

$$
N^{n / 2}|\Delta|=2^{n(n-1) / 2} \prod_{k=1}^{n-1}|\sin (k x)|^{n-k} .
$$

On the other hand,

$$
\begin{aligned}
\prod_{k=1}^{n-1} \sin ^{n-k}(k x) & =\prod_{k=1}^{n-1}\left(\frac{\sin (k x)}{\sin (x)}\right)^{n-k} \sin ^{n-k}(x) \\
& =\prod_{k=1}^{n-1} U_{k-1}^{n-k}(\cos x) \sin ^{n-k}(x)
\end{aligned}
$$

where $U_{k}(x)$ denotes the Chebyshev polynomial of the second kind and degree $k$, normalized so that $U_{k-1}(1)=k$.
Proceeding,

$$
\prod_{k=1}^{n-1} \sin ^{n-k}(k x)=(\sin x)^{n(n-1) / 2} \prod_{k=1}^{n-1} U_{k-1}^{n-k}(\cos x),
$$

and so

$$
\prod_{k=1}^{n-1} \sin ^{n-k}(k x)=(\sin x)^{n(n-1) / 2} P_{m}(\cos x)
$$

where $P_{m}$ is a certain polynomial of degree

$$
m=\sum_{i=1}^{n-2} i(n-i-1)=\frac{n(n-1)(n-2)}{6} .
$$

The bound

$$
N^{n / 2}|\Delta| \leq 2^{n(n-1) / 2} C(n) \sin \left(\frac{\pi}{N}\right)^{n(n-1) / 2}
$$

where

$$
C(n)=1 ! 2 ! 3 ! 4 ! \cdots(n-1) !
$$

is therefore asymptotically correct (the cosine terms have been neglected). A further approximation is

$$
N^{n / 2}|\Delta| \approx 2^{n(n-1) / 2} C(n)\left(\frac{\pi}{N}\right)^{n(n-1) / 2} .
$$

Since the determinant $\Delta$ is the product of the eigenvalues $\lambda$, one has

$$
\left|\lambda_{\min }\right|^{n} \leq|\Delta| \leq\left|\lambda_{\max }\right|^{n}
$$

where $\lambda_{\min }$ and $\lambda_{\max }$ denote the smallest and largest eigenvalues in absolute value. This shows that the minimum eigenvalue of the matrix satisfies

$$
\left|\lambda_{\min }\right| \leq|\Delta|^{1 / n} \leq \frac{C(n)^{1 / n}}{\sqrt{N}}\left[2 \sin \left(\frac{\pi}{N}\right)\right]^{(n-1) / 2} .
$$

Note that $\lambda_{\min }$ decreases as $N$ increases, even if the total number of unknown samples $n$ remains constant. It is possible to estimate $C(n)$ using the Euler-MacLaurin summation formula. This leads to

$$
C(n)^{1 / n} \approx \exp \left(\frac{n}{2} \log (n)-\frac{3 n}{4}+1\right)
$$

Usually, $n$ is much smaller than $N$, and the sin can be replaced by its argument. It follows that $\lambda_{\min }$ is bounded by a quantity which is $O\left(\sqrt{n} e^{-3 n / 4}\right)$ times $O\left[(\pi \sqrt{n} / N)^{n}\right]$. The matrix $A$ has, in general, very small eigenvalues and may become singular to machine precision even for moderate values of $n$ and $N$ (say, $n=10$ and $N=100$ ). 


\section{OTHER METHODS}

The matrices $A A^{H}$ or $A^{H} A$ have the same (real) nonzero eigenvalues, and it is well known that they are the squares of the nonzero singular values of $A$. It is clear that

$$
\left(A A^{H}\right)_{a b}=\sum_{j=0}^{n-1} F_{i_{a} j} F_{j i_{b}}^{*}=\frac{1}{N} \sum_{j=0}^{n-1} w^{\left(i_{a}-i_{b}\right) j},
$$

and

$$
\left(A^{H} A\right)_{a b}=\sum_{j=0}^{n-1} F_{a i_{j}}^{*} F_{i_{j} b}=\frac{1}{N} \sum_{j=0}^{n-1} w^{-i_{j}(a-b)} .
$$

But these equations are similar to those that define the matrices of the direct methods studied in $[3,10,17]$. Therefore, the singular values of $A$ are the square roots of the eigenvalues of $A A^{H}$ or $A^{H} A$. These eigenvalues have been studied (a recent reference is [6]), and the results can immediately be applied to $A$ as well.

\section{REFERENCES}

[1] J. A. Cadzow. An extrapolation procedure for bandlimited signals. IEEE Trans. Acoust. Speech Signal Processing, 27(1):4-12, Feb. 1979.

[2] P. J. S. G. Ferreira. Interpolation and the discrete Papoulis-Gerchberg algorithm. IEEE Trans. Signal Processing, 42(10):2596-2606, Oct. 1994.

[3] P. J. S. G. Ferreira. Noniterative and faster iterative methods for interpolation and extrapolation. IEEE Trans. Signal Processing, 42(11):3278-3282, Nov. 1994.

[4] P. J. S. G. Ferreira. The stability of certain image restoration problems: quantitative results. In: Proceedings of the Second IEEE International Conference on Image Processing, ICIP-95, volume II, pp. 29-32, Washington, D.C., U.S.A., Oct. 1995.

[5] P. J. S. G. Ferreira. Interpolation in the time and frequency domains. IEEE Sig. Proc. Letters, 3(6):176178, Jun. 1996.

[6] P. J. S. G. Ferreira. The eigenvalues of matrices which occur in certain interpolation problems. IEEE Trans. Signal Processing, 45(8):2115-2120, Aug. 1997.

[7] P. J. S. G. Ferreira and A. J. Pinho. Errorless restoration algorithms for band-limited images. In: Proceedings of the First IEEE International Conference on Image Processing, ICIP-94, volume III, pp. 157-161, Austin, TX, U.S.A., Nov. 1994.
[8] R. W. Gerchberg. Super-resolution through error energy reduction. Optica Acta, 21(9):709-720, 1974.

[9] M. Greitans. Iterative reconstruction of lost samples using updating of autocorrelation matrix. In: Proceedings of the 1997 Workshop on Sampling Theory and Applications, SampTA-97, pp. 155-160, Aveiro, Portugal, Jun. 1997.

[10] K. Gröchenig. A discrete theory of irregular sampling. Linear Algebra Appl., 193:129-150, 1993.

[11] M. C. Jones. The discrete Gerchberg algorithm. IEEE Trans. Acoust. Speech Signal Processing, 34(3):624626, June 1986.

[12] F. Marvasti, M. Echhart, and M. Hasan. Burst correction for missing samples. In: Proceedings of the 1997 Workshop on Sampling Theory and Applications, SampTA-97, pp. 161-167, Aveiro, Portugal, June 1997.

[13] A. Papoulis. A new algorithm in spectral analysis and band-limited extrapolation. IEEE Trans. Circuits Syst., 22(9):735-742, Sep. 1975.

[14] M. I. Sezan and H. Stark. Image restoration by the method of convex projections: Part 2 - applications and numerical results. IEEE Trans. Med. Imag., 1(2):95-101, Oct. 1982.

[15] D. Slepian and H. O. Pollak. Prolate spheroidal wave functions, Fourier analysis and uncertainty - I. Bell Syst. Tech. J., 40(1):43-63, Jan. 1961.

[16] T. Strohmer. Efficient Methods for Digital Signal and Image Reconstruction from Nonuniform Samples. Ph.D. Thesis, Institut für Mathematik der Universität Wien, Nov. 1993.

[17] T. Strohmer. On discrete band-limited signal extrapolation. In: M. E. H. Ismail, M. Z. Nashed, A. I. Zayed, and A. F. Ghaleb, editors, Mathematical Analysis, Wavelets, and Signal Processing, Vol. 190 of Contemporary Mathematics, pp. 323-337, Providence, Rhode Island, 1995 (American Mathematical Society).

[18] D. O. Walsh and P. A. Nielsen-Delaney. Direct method for superresolution. J. Opt. Soc. Am. A, 11(2):572579, Feb. 1994.

[19] D. C. Youla. Generalized image restoration by the method of alternating orthogonal projections. IEEE Trans. Circuits Syst., 25(9):694-702, Sep. 1978.

[20] D. C. Youla and H. Webb. Image restoration by the method of convex projections: Part 1 - theory. IEEE Trans. Med. Imag., 1(2):81-94, Oct. 1982. 